\title{
The Construction of Pairwise Additive Minimal BIB Designs with Asymptotic Results
}

\author{
Kazuki Matsubara', Sanpei Kageyama ${ }^{2}$ \\ ${ }^{1}$ Graduate School of Science, Hiroshima University, Higashi-Hiroshima, Japan \\ ${ }^{2}$ Hiroshima Institute of Technology, Hiroshima, Japan \\ Email: d122307@hiroshima-u.ac.jp, s.kageyama.4b@it-hiroshima.ac.jp
}

Received 21 April 2014; revised 28 May 2014; accepted 12 June 2014

Copyright (C) 2014 by authors and Scientific Research Publishing Inc.

This work is licensed under the Creative Commons Attribution International License (CC BY). http://creativecommons.org/licenses/by/4.0/

\section{(c) (7) Open Access}

\begin{abstract}
An asymptotic existence of balanced incomplete block (BIB) designs and pairwise balanced designs (PBD) has been discussed in [1]-[3]. On the other hand, the existence of additive BIB designs and pairwise additive BIB designs with $k=2$ and $\lambda=1$ has been discussed with direct and recursive constructions in [4]-[8]. In this paper, an asymptotic existence of pairwise additive BIB designs is proved by use of Wilson's theorem on PBD, and also for some $\ell$ and $k$ the exact existence of $\ell$ pairwise additive BIB designs with block size $k$ and $\lambda=1$ is discussed.
\end{abstract}

\section{Keywords}

Incidence Matrix, Pairwise Balanced Design (PBD), Balanced Incomplete Block Design (BIBD), Additive BIB Design, Pairwise Additive BIB Design, Wilson's Theorem

\section{Introduction}

A pairwise balanced design (PBD) of order $v$ with block sizes in a set $K$ is a system $(V, \mathcal{B})$, where $V$ is a finite set (the point set) of cardinality $v$ and $\mathcal{B}$ is a family of subsets (blocks) of $V$ such that 1 ) if $B \in \mathcal{B}$, then $|B| \in K$ and 2) every pair of distinct elements of $V$ occurs in $\lambda$ blocks of $\mathcal{B}$ [9]. This is denoted by $\operatorname{PBD}(v, K, \lambda)$. When $K=\{k\}$, a $\operatorname{PBD}(v,\{k\}, \lambda)$ is especially called a balanced incomplete block (BIB) design, where $|\mathcal{B}|=b$, each block contains $k$ different points and each point appears in $r$ different blocks [10]. This is denoted by $\operatorname{BIBD}(v, b, r, k, \lambda)$ or $\mathrm{B}(v, k, \lambda)$. It is well known that necessary conditions for the existence of a $\mathrm{B}(v, k, \lambda)$ are 


$$
\lambda(v-1) \equiv 0(\bmod k-1), \lambda v(v-1) \equiv 0(\bmod k(k-1)) .
$$

Let $N=\left(n_{i j}\right)$ be the $v \times b$ incidence matrix of a BIB design, where $n_{i j}=1$ or 0 for all $i(=1,2, \cdots, v)$ and $j(=1,2, \cdots, b)$, according as the $i$-th point occurs in the $j$-th block or otherwise. Hence the incidence matrix $N$ satisfies the conditions: 1) $\sum_{j=1}^{b} n_{i j}=r$ for all $i$, 2) $\sum_{i=1}^{v} n_{i j}=k$ for all $j$, 3) $\sum_{j=1}^{b} n_{i j} n_{i^{\prime} j}=\lambda$ for all $i, i^{\prime}\left(i \neq i^{\prime}\right)=1,2, \cdots, v$.

Let $s=v / k$, where $s$ need not be an integer unlike other parameters. Further let $2 \leq \ell \leq s$. A set of $\ell$ $\operatorname{BIBD}(v, b, r, k, \lambda)$ is called $\ell$ pairwise additive BIB designs if $\ell$ corresponding incidence matrices $N_{1}, N_{2}, \ldots, N_{\ell}$ of the BIB design satisfy that $\boldsymbol{N}_{i_{1}}+\boldsymbol{N}_{i_{2}}$ is the incidence matrix of a $\operatorname{BIBD}\left(v^{*}=v=s k, b^{*}=b, r^{*}=2 r, k^{*}=2 k^{\ell}\right.$, $\left.\lambda^{*}=2 r(2 k-1) /(s k-1)\right)$ for any distinct $i_{1}, i_{2} \in\{1,2, \cdots, \ell\}$. When $\ell=s$, this is especially called additive BIB designs [6] [7].

It is clear by the definition that the existence of $\ell$ pairwise additive $\mathrm{B}(v, k, \lambda)$ implies the existence of $\ell^{\prime}$ pairwise additive $\mathrm{B}(v, k, \lambda)$ for any $\ell^{\prime}<\ell$. Hence, for given parameters $v, k, \lambda$, the larger $\ell$ is, the more difficult a construction problem of $\ell$ pairwise additive BIB designs is.

In pairwise additive $\mathrm{B}(v, k, \lambda)$, since a sum of any two incidence matrices yields a BIB design, it is seen [7] that

$$
\frac{2 \lambda}{k-1} \text { is a positive integer. }
$$

It follows from (1.2) that the existence of $\ell$ pairwise additive $\mathrm{B}(v, k, \lambda)$ implies

$$
\lambda \geq \begin{cases}(k-1) / 2 & (k \text { is odd }), \\ k-1 & (k \text { is even }) .\end{cases}
$$

Pairwise additive $\mathrm{B}(v, k, \lambda)$ are said to be minimal if $\lambda=(k-1) / 2$ or $k-1$ according as $k$ is odd or even.

Some classes of $\ell$ pairwise additive $\mathrm{B}(v, k, \lambda)$ are constructed in [4]-[8]. It is clear by the definition that $v \geq \ell k$. The purpose of this paper is to show that, for a given odd prime power $k$ and a given positive integer $\ell(\leq k)$, the necessary conditions (1.1) for the existence of $\ell$ pairwise additive minimal $\mathrm{B}(v, k, \lambda=(k-1) / 2)$ are asymptotically sufficient on $v(\geq \ell k)$. In particular, for the existence of $\ell$ pairwise additive minimal $\mathrm{B}(v, 3,1)$, (1.1) is asymptotically sufficient, i.e., there are $\ell$ pairwise additive minimal $\mathrm{B}(v, 3,1)$ for sufficiently larger $v \equiv 1,3(\bmod 6)$, even if $\ell>k$. Furthermore, as the exact existence, it is shown that there are 2 pairwise additive $\mathrm{B}(6 m+1,3,1)$ for any positive integer $m$ except possibly for 12 values.

\section{2. $\operatorname{PBD}(v, K, \lambda)$}

The existence of $\operatorname{PBD}(v, K, \lambda)$ is reviewed along with necessary and asymptotically sufficient conditions.

Let $K$ be a set of positive integers and

$$
\alpha(K)=\operatorname{gcd}\{k-1 \mid k \in K\}, \quad \beta(K)=\operatorname{gcd}\{k(k-1) \mid k \in K\} .
$$

Necessary conditions for the existence of a $\operatorname{PBD}(v, K, \lambda)$ are known as follows.

Lemma 2.1 [2] Necessary conditions for the existence of a $\operatorname{PBD}(v, K, \lambda)$ are

$$
\lambda(v-1) \equiv 0(\bmod \alpha(K)), \lambda v(v-1) \equiv 0(\bmod \beta(K)) .
$$

Wilson [3] proved the asymptotic existence as Theorem 2.2 below shows.

Theorem 2.2 The necessary conditions (2.1) for the existence of a $\operatorname{PBD}(v, K, \lambda)$ are asymptotically sufficient.

For any set $K$ of positive integers and any positive integer $\lambda$, let $c(K, \lambda)$ denote the smallest integer such that there are $\operatorname{PBD}(v, K, \lambda)$ for every integer $v(\geq c(K, \lambda))$ satisfying (2.1). Then Theorem 2.2 states the existence of $c(K, \lambda)$. On the other hand, some explicit bound for $c(K, \lambda)$ was provided as follows.

Lemma $2.3[11]$ There are $\operatorname{PBD}(v,\{8,9,10\}, 1)$ for all positive integers $v \geq 583$.

Especially, for a set $P_{1,6}$ being a set of prime powers of form $6 m+1, \quad c\left(P_{1,6}, 1\right) \leq 1321$ is shown as follows.

Lemma 2.4 ([12] Theorem 19.69) Let $P_{1,6}$ be a set of prime powers of form $6 m+1$ with a positive integer $m$. Then there are $\operatorname{PBD}\left(v, P_{1,6}, 1\right)$ for all positive integers $v \equiv 1(\bmod 6)$, except possibly for $6 m+1 \in$ $\{55,115,145,205,235,265,319,355,391,415,445,451,493,649,667,685,697,745,781,799,805,1315\}$. 


\section{Construction by $\operatorname{PBD}(v, K, \lambda)$}

In this section, a method of constructing pairwise additive $\operatorname{BIB}$ designs through $\operatorname{PBD}(v, K, \lambda)$ is provided.

The following simple method is useful to construct pairwise additive BIB designs.

Lemma 3.1 The existence of a $\operatorname{PBD}(v, K, \lambda)$ and $\ell$ pairwise additive $\mathrm{B}\left(v^{\prime}=k, k^{\prime}, \lambda^{\prime}\right)$ for any $k \in K$ implies the existence of $\ell$ pairwise additive $\mathrm{B}\left(v, k^{\prime}, \lambda \lambda^{\prime}\right)$.

Proof. Let $(V, \mathcal{B})$ be the $\operatorname{PBD}(v, K, \lambda)$ and $B_{i} \in \mathcal{B}, 1 \leq i \leq|\mathcal{B}|$. On the set $B_{i}$, let a block set $B_{i}$ with all block size $k^{\prime}$ be formed by the $\ell$ pairwise additive $\mathrm{B}\left(v^{\prime}=k, k^{\prime}, \lambda^{\prime}\right)$ for each $i$. Then it follows that the $\left(V, \bigcup_{i=1}^{|\mathcal{B}|} \mathcal{B}_{i}\right)$ is the required BIB design. $\square$

For example, Lemma 3.1 yields the following.

Theorem 3.2 There are 4 pairwise additive $\mathrm{B}(v, 2,1)$ for any integer $v(\geq 583)$.

Proof. It follows from the fact ([4] [6]) that there are additive $B(8,2,1), 4$ pairwise additive $B(9,2,1)$ and additive $B(10,2,1)$. Hence Lemmas 2.3 and 3.1 can yield the required designs.

As the next case of block sizes, $k=3$ is considered. A concept of $2 m$ pairwise additive $\mathrm{B}(6 m+1,3,1)$ has been discussed as a compatibly nested minimal partition in [12], which shows the existence of pairwise additive $\mathrm{B}(6 m+1,3,1)$ as follows.

Lemma 3.3 ([12]; Theorem 22.12) Let $6 m+1$ be an odd prime power for a positive integer $m$. Then there are $2 m$ pairwise additive $\mathrm{B}(6 m+1,3,1)$.

Lemmas 2.4, 3.1 and 3.3 can produce the following.

Theorem 3.4 ([12]; Theorem 22.13) There are 2 pairwise additive $\mathrm{B}(6 m+1,3,1)$ for all positive integers $m$, except possibly for $6 m+1 \in\{55,115,145,205,235,265,319,355,391,415,445,451,493,649,667,685,697,745$, $781,799,805,1315\}$.

Theorem 3.4 will be improved as in Theorem 6.7.

\section{Some Class of Pairwise Additive $\mathrm{B}(v, k,(k-1) / 2)$}

In this section, a necessary condition for the existence of pairwise additive $\mathrm{B}(v, k,(k-1) / 2)$ being minimal is provided and then some classes of $(v-1) / k$ pairwise additive $\mathrm{B}(v, k,(k-1) / 2)$ and $(k$ pairwise) additive $\mathrm{B}\left(v=k^{2}, k,(k-1) / 2\right)$ are constructed.

Now (1.1) implies that necessary conditions for the existence of pairwise additive $\mathrm{B}(v, k,(k-1) / 2)$ are

$$
v-1 \equiv 0(\bmod 2), v(v-1) \equiv 0(\bmod 2 k) .
$$

Furthermore, the following is given.

Theorem 4.1 When $k$ is an odd prime power, necessary conditions for the existence of pairwise additive $\mathrm{B}(v, k,(k-1) / 2)$ are

$$
v \equiv 1, k(\bmod 2 k) .
$$

Proof. Since $v(v-1) \equiv 0(\bmod 2 k)$ and $\operatorname{gcd}(v, v-1)=1$, when $k$ is an odd prime power, it is shown that either $v \equiv 0$ or $v-1 \equiv 0(\bmod k)$. Hence $v-1 \equiv 0(\bmod 2)$ implies $v \equiv 1, k(\bmod 2 k)$.

When $k$ is an odd prime power, a class of pairwise additive $\mathrm{B}(v, k,(k-1) / 2)$ is obtained as follows. This observation shows a generalization of Lemma 3.3.

Theorem 4.2 Let both $2 k m+1$ and $k$ be odd prime powers for a positive integer $m$. Then there are $2 m(=(v-1) / k)$ pairwise additive $\mathrm{B}(2 k m+1, k,(k-1) / 2)$.

Proof. It can be shown that a development of the following initial blocks on $\mathrm{GF}(2 \mathrm{~km}+1)$ yields incidence matrices $N_{1}, N_{2}, \cdots, N_{2 m}$ of the required BIB design:

$$
\begin{aligned}
N_{i}: & \left\{\alpha^{i}, \alpha^{2 m+i}, \alpha^{4 m+i}, \cdots, \alpha^{2(k-1) m+i}\right\}, \\
& \left\{\alpha^{i+1}, \alpha^{2 m+i+1}, \alpha^{4 m+i+1}, \cdots, \alpha^{2(k-1) m+i+1}\right\}, \\
& \vdots \\
& \left\{\alpha^{i+m-1}, \alpha^{3 m+i-1}, \alpha^{5 m+i-1}, \cdots, \alpha^{(2 k-1) m+i-1}\right\},
\end{aligned}
$$

where $\alpha$ is a primitive element of $\operatorname{GF}(2 k m+1)$ and $1 \leq i \leq 2 m$. 
Furthermore, the following is known to be provided by recursive constructions with affine resolvable BIB designs. This result will be used in the next section.

Theorem 4.3 [7] Let $k$ be an odd prime power. Then there are additive $\mathrm{B}\left(k^{2}, k,(k-1) / 2\right)$.

Especially, when $k=3$, the further result is known.

Theorem 4.4 [8] There are additive $\mathrm{B}\left(3^{n}, 3,1\right)$ for any positive integer $n(\geq 2)$.

\section{Asymptotic Existence of Pairwise Additive Minimal $\mathrm{B}(v, k,(k-1) / 2)$}

In this section, when $k$ is an odd prime power, an asymptotic existence of pairwise additive $\mathrm{B}(v, k,(k-1) / 2)$ is discussed, and it is shown that the necessary conditions (4.1) for the existence of $\ell$ pairwise additive $\mathrm{B}(v, k,(k-1) / 2)$ are asymptotically sufficient for a given positive integer $\ell(\leq k)$.

Dirichlet's theorem on primes is useful for the present discussion.

Theorem 5.1 (Dirichlet) If $\operatorname{gcd}(a, b)=1$, then a set of integers of the following form

$$
a n+b, n=1,2, \cdots
$$

contains infinitely many primes.

Now Theorem 5.1 yields the following.

Lemma 5.2 [13] For any positive even integer $m$, there are primes $p$ and $q$ for which $p \equiv q \equiv 1(\bmod m)$ and $\operatorname{gcd}(p(p-1), q(q-1))=m$.

In the proof of Lemma 5.2 (i.e., Lemma 3.4 in [13]), primes $p$ and $q$ are obtained by using Theorem 5.1. Thus Lemma 5.2 implies the existence of sufficiently large primes $p$ and $q$ as follows.

Lemma 5.3 For a given odd prime power $k$, there are primes $p$ and $q$ such that (a) $p>q>k^{2}$, (b) $p \equiv q \equiv 1(\bmod 2 k)$, (c) $\alpha(K)=2$ and (d) $\beta(K)=2 k$ for $K=\{p, q\} \cup\left\{k^{2}\right\}$.

Proof. Let $k$ be an odd prime power. Then, for an even integer $2 k$, Lemma 5.2 provides primes $p$ and $q$ such that (a) $p>q>k^{2}$, (b) $p \equiv q \equiv 1(\bmod 2 k)$ and $\operatorname{gcd}(p(p-1), q(q-1))=2 k$. Hence it is seen that $\operatorname{gcd}(p-1, q-1)=2 k, \operatorname{gcd}\left(2 k, k^{2}-1\right)=2$ and $\operatorname{gcd}\left(2 k, k^{2}\left(k^{2}-1\right)\right)=2 k$.

Now let $K=\{p, q\} \cup\left\{k^{2}\right\}$. Then

$$
\begin{aligned}
& \alpha(K)=\operatorname{gcd}\left\{p-1, q-1, k^{2}-1\right\}=2, \\
& \beta(K)=\operatorname{gcd}\left\{p(p-1), q(q-1), k^{2}\left(k^{2}-1\right)\right\}=2 k,
\end{aligned}
$$

which imply (c) and (d). 口

Thus one of the main results of this paper is now obtained through conditions (a), (b), (c) and (d) given in Lemma 5.3.

Theorem 5.4 For a given odd prime power $k,(4.1)$ is a necessary and asymptotically sufficient condition for the existence of $k$ pairwise additive $\mathrm{B}(v, k,(k-1) / 2)$.

Proof (sufficiency). Let $p$ and $q$ be primes as in Lemma 5.3 with $K=\{p, q\} \cup\left\{k^{2}\right\}$. Then conditions (c) and (d) show that there are $\operatorname{PBD}(v, K, 1)$ for sufficiently large $v$ satisfying (4.1), on account of Theorem 2.2. Conditions (a) and (b) show that there are $(p-1) / k(\geq k)$ pairwise additive $\mathrm{B}(p, k,(k-1) / 2)$, $(q-1) / k(\geq k)$ pairwise additive $\mathrm{B}(q, k,(k-1) / 2)$ and additive $\mathrm{B}\left(k^{2}, k,(k-1) / 2\right)$, on account of Theorems 4.2 and 4.3. Hence the required designs can be obtained on account of Lemma 3.1. 口

Unfortunately, by use of Theorem 5.4 we cannot show the existence of $\ell$ pairwise additive $\mathrm{B}(v, k,(k-1) / 2)$ for $\ell>k$, since an additive $\mathrm{B}\left(k^{2}, k,(k-1) / 2\right)$ means $k$ pairwise additive $\mathrm{B}\left(k^{2}, k,(k-1) / 2\right)$.

Next, for a given odd prime power $k$ and a given positive integer $\ell$, even if $\ell>k$, the existence of $\ell$ pairwise additive $\mathrm{B}(v, k,(k-1) / 2)$ is discussed for sufficiently large $v \equiv 1(\bmod 2 k)$.

Lemma 5.5 For a given odd prime power $k$ and a given positive integer $\ell$, there are primes $p$ and $q$ such that (a) $p>q>k \ell$, (b) $p \equiv q \equiv 1(\bmod 2 k)$ and (c) $\alpha(\{p, q\})=\beta(\{p, q\})=2 k$.

Proof. Let $k$ be an odd prime power and $\ell$ be a positive integer. Then, for a positive integer $2 k$, Lemma 5.2 provides primes $p$ and $q$ such that (a) $p>q>k \ell$, (b) $p \equiv q \equiv 1(\bmod 2 k)$ and $\operatorname{gcd}(p(p-1), q(q-1))=2 k$. 
Hence it is seen that $\operatorname{gcd}(p-1, q-1)=2 k$ and (c) holds.

Thus the following result is obtained through conditions (a), (b) and (c) as in Lemma 5.5.

Theorem 5.6 For a given odd prime power $k$ and a given positive integer $\ell$, there are $\ell$ pairwise additive $\mathrm{B}(v, k,(k-1) / 2)$ for sufficiently large $v \equiv 1(\bmod 2 k)$.

Proof. Let $p$ and $q$ be primes as in Lemma 5.5. Then it follows from (c) that there are $\operatorname{PBD}(v,\{p, q\}, 1)$ for sufficiently large $v \equiv 1(\bmod 2 k)$, on account of Theorem 2.2. Also Theorem 4.2 along with conditions (a) and (b) shows that there are $(p-1) / k(\geq \ell)$ pairwise additive $\mathrm{B}(p, k,(k-1) / 2)$ and $(q-1) / k(\geq \ell)$ pairwise additive $\mathrm{B}(q, k,(k-1) / 2)$. Thus the required designs are obtained on account of Lemma 3.1.

\section{Pairwise Additive $\mathrm{B}(v, 3,1)$}

In this section, the existence of pairwise additive $\mathrm{B}(v, 3,1)$ is discussed. At first it is shown that there are $\ell$ pairwise additive $\mathrm{B}(v, 3,1)$ for sufficiently large $v \equiv 1,3(\bmod 6)$, even if $\ell>k$. Furthermore, the exact existence of 2 pairwise additive $\mathrm{B}(v, 3,1)$ with $v \equiv 1,3(\bmod 6)$ is discussed by providing direct and recursive constructions of pairwise additive $\mathrm{B}(v, 3,1)$. Finally, it is shown that there are 2 pairwise additive $\mathrm{B}(v, 3,1)$ for any $v \equiv 1(\bmod 6)$ except possibly for 12 values.

Three classes of pairwise additive $\mathrm{B}(v, 3,1)$ are given as in Lemma 3.3 and Theorems 3.4 and 4.4. For $v \equiv 1,3(\bmod 6), 15$ is the smallest value of $v$ for which the existence of 2 pairwise additive $\mathrm{B}(v, 3,1)$ is unknown in literature. Hence at first this case is individually considered here.

Lemma 6.1 There are 2 pairwise additive $\mathrm{B}(15,3,1)$.

Proof. It can be shown that a development of the following initial blocks on $Z_{7}$ with the index being fixed yields incidence matrices $N_{1}, N_{2}$ of the required BIB design:

$$
\begin{aligned}
& N_{1}:\left\{0_{0}, 1_{1}, 6_{1}\right\},\left\{0_{0}, 2_{1}, 5_{1}\right\},\left\{0_{0}, 3_{1}, 4_{1}\right\},\left\{1_{0}, 2_{0}, 4_{0}\right\},\left\{0_{0}, 0_{1}, \infty\right\} \bmod 7 \\
& N_{2}:\left\{0_{1}, 2_{0}, 5_{0}\right\},\left\{0_{1}, 3_{0}, 4_{0}\right\},\left\{0_{1}, 1_{0}, 6_{0}\right\},\left\{0_{0}, 0_{1}, \infty\right\},\left\{1_{1}, 2_{1}, 4_{1}\right\} \bmod 7
\end{aligned}
$$

with 15 elements $\left\{i_{j} \mid i \in Z_{7}, j \in Z_{2}\right\} \cup\{\infty\}$. Here, in general $Z_{s}=\{0,1, \cdots, s-1\}$.

Now, $\ell$ pairwise additive $\mathrm{B}(v, 3,1)$ with sufficiently large $v \equiv 1,3(\bmod 6)$ are obtained as follows. This shows an extension of Theorem 5.4 with $k=3$.

Theorem 6.2 For a given positive integer $\ell$, even if $\ell>3$, there are $\ell$ pairwise additive $\mathrm{B}(v, 3,1)$ with sufficiently large $v \equiv 1,3(\bmod 6)$.

Proof. Let $n$ be a positive integer satisfying $3^{n} \geq 3 \ell$. Then $\left(3^{n-1}\right.$ pairwise $)$ additive $\mathrm{B}\left(3^{n}, 3,1\right)$ are constructed by Theorem 4.4, and there are primes $p$ and $q$ such that $p>q>3 \ell, p \equiv q \equiv 1(\bmod 6)$ and $\operatorname{gcd}(p(p-1), q(q-1))=6$, on account of Lemma 5.2. Furthermore, since $\alpha(K)=2$ and $\beta(K)=6$ with $K=\{p, q\} \cup\left\{3^{n}\right\}$, there are $\operatorname{PBD}\left(v,\left\{p, q, 3^{n}\right\}, 1\right)$ for sufficiently large $v \equiv 1,3(\bmod 6)$, on account of Theorem 2.2. Hence $\ell$ pairwise additive $\mathrm{B}(v, 3,1)$ for sufficiently large $v \equiv 1,3(\bmod 6)$ can be constructed by Lemma 3.1 with $(p-1) / 3(\geq \ell)$ pairwise additive $\mathrm{B}(p, 3,1),(q-1) / 3(\geq \ell)$ pairwise additive $\mathrm{B}(q, 3,1)$ and additive $\mathrm{B}\left(3^{n}, 3,1\right)$.

Next, some recursive constructions of pairwise additive $\mathrm{B}(v, 3,1)$ are provided. A combinatorial structure is here introduced. A transversal design, denoted by $\operatorname{TD}_{\lambda}(k, n)$, is a triple $(V, \mathcal{G}, \mathcal{B})$ such that 1$) V$ is a set of $k n$ elements, 2) $\mathcal{G}$ is a partition of $V$ into $k$ classes (groups), each of size $n, 3$ ) $\mathcal{B}$ is a family of $k$-subsets (blocks) of $V, 4$ ) every unordered pair of elements from the same group is not contained in any block, and 5) every unordered pair of elements from other groups is contained in exactly $\lambda$ blocks. When $\lambda=1$, we simply write $\operatorname{TD}(k, n)$, where $|\mathcal{B}|=n^{2}$ [14].

Since it is known [14] that the existence of a $\operatorname{TD}(k+2, n)$ is equivalent to the existence of $k$ mutually orthogonal latin squares of order $n$, the following result can be obtained, when $k=4$.

Lemma 6.3 [14] There exists a $\operatorname{TD}(6, n)$ for all $n(\geq 5)$ except for $n=6$ and possibly for $n \in\{10,14,18,22\}$. A method of construction is presented, similarly to a recursive construction given in [4], by use of $\operatorname{TD}(6, n)$.

Theorem 6.4 The existence of $\ell$ pairwise additive $\mathrm{B}(v, 3,1), \ell$ pairwise additive $\mathrm{B}\left(v^{\prime}, 3,1\right)$ and $a$ $\mathrm{TD}(3 \ell, v)$ implies the existence of $\ell$ pairwise additive $\mathrm{B}\left(v v^{\prime}, 3,1\right)$. 
Proof. Let $\mathcal{B}_{h}, \mathcal{B}_{h}^{\prime}, 1 \leq h \leq \ell$, be block sets of $\ell$ pairwise additive $\mathrm{B}(v, 3,1)$ and $\ell$ pairwise additive $\mathrm{B}\left(v^{\prime}, 3,1\right)$ respectively as

$$
\begin{aligned}
& \mathcal{B}_{h}=\left\{\left\{x_{i}^{(h)}, y_{i}^{(h)}, z_{i}^{(h)}\right\} \mid 1 \leq i \leq \frac{v(v-1)}{6}\right\}, \\
& \mathcal{B}_{h}^{\prime}=\left\{\left\{x_{j}^{\prime(h)}, y_{j}^{(h)}, z_{j}^{\prime(h)}\right\} \mid 1 \leq j \leq \frac{v^{\prime}\left(v^{\prime}-1\right)}{6}\right\} .
\end{aligned}
$$

and let $d(m, n), 1 \leq m \leq v^{2}$ and $1 \leq n \leq 3 \ell$, denote an element which occurs in both the $m$-th block of a $\operatorname{TD}(3 \ell, v)$ and the $n$-th group. Then it can be shown that the following $\ell$ incidence matrices yield the required $\ell$ pairwise additive BIB designs with $v v^{\prime}$ elements denoted by $(s, t)$ for $1 \leq s \leq v$ and $1 \leq t \leq v^{\prime}$ :

$$
\begin{aligned}
N_{h}:\left\{\left(x_{i}^{(h)}, t\right),\left(y_{i}^{(h)}, t\right),\left(z_{i}^{(h)}, t\right)\right\}, \\
\quad\left\{\left(d(m, 3 h-2), x_{j}^{(h)}\right),\left(d(m, 3 h-1), y_{j}^{(h)}\right),\left(d(m, 3 h), z_{j}^{\prime(h)}\right)\right\}
\end{aligned}
$$

where $1 \leq h \leq \ell, 1 \leq i \leq v(v-1) / 6,1 \leq j \leq v^{\prime}\left(v^{\prime}-1\right) / 6,1 \leq t \leq v^{\prime}$ and $1 \leq m \leq v^{2}$.

Another recursive method is presented.

Theorem 6.5 The existence of $\ell$ pairwise additive $\mathrm{B}(v+1,3,1), \ell$ pairwise additive $\mathrm{B}\left(v^{\prime}, 3,1\right)$ and a $\mathrm{TD}(3 \ell, v)$ implies the existence of $\ell$ pairwise additive $\mathrm{B}\left(v v^{\prime}+1,3,1\right)$.

Proof. Let $\mathcal{B}_{h}^{\prime}, 1 \leq h \leq \ell$, be a block set similarly to the proof of Theorem 6.4 and let $\mathcal{B}_{h}, 1 \leq h \leq \ell$, be a block set of $\ell$ pairwise additive $\mathrm{B}(v+1,3,1)$, where

$$
\mathcal{B}_{h}=\left\{\left\{x_{i}^{(h)}, y_{i}^{(h)}, z_{i}^{(h)}\right\} \mid 1 \leq i \leq \frac{v(v+1)}{6}\right\},
$$

with $v+1$ elements $1,2, \cdots, v$ and $\infty$. Also let $d(m, n), 1 \leq m \leq v^{2}$ and $1 \leq n \leq 3 \ell$, denote an element which occurs in both the $m$-th block of a $\operatorname{TD}(3 \ell, v)$ and the $n$-th group. Then the following $\ell$ incidence matrices can yield the required $\ell$ pairwise additive BIB designs with $v v^{\prime}+1$ elements denoted by $(s, t)$ for $1 \leq s \leq v$ and $1 \leq t \leq v^{\prime}$, and $\infty(=(\infty, t))$ :

$$
\begin{aligned}
N_{h}:\left\{\left(x_{i}^{(h)}, t\right),\left(y_{i}^{(h)}, t\right),\left(z_{i}^{(h)}, t\right)\right\}, \\
\quad\left\{\left(d(m, 3 h-2), x_{j}^{(h)}\right),\left(d(m, 3 h-1), y_{j}^{(h)}\right),\left(d(m, 3 h), z_{j}^{(h)}\right)\right\},
\end{aligned}
$$

where $1 \leq h \leq \ell, 1 \leq i \leq v(v+1) / 6,1 \leq j \leq v^{\prime}\left(v^{\prime}-1\right) / 6,1 \leq t \leq v^{\prime}$ and $1 \leq m \leq v^{2}$.

Now 2 pairwise additive $\mathrm{B}(v, 3,1)$ are more obtained.

Lemma 6.6 There are 2 pairwise additive $\mathrm{B}(v, 3,1)$ for $v \in\{235,391,445,451,649,685,745,781,799,805\}$.

Proof. For $v=235,391,445,451,649,685,745,781,799,805$, Theorem 6.5 with

$$
\left(v, v^{\prime}\right)=(26,9),(30,13),(12,37),(30,15),(8,81),(36,19),(24,31),(60,13),(42,19),(12,67)
$$

provides the required BIB designs respectively, because 2 pairwise additive $\mathrm{B}(v+1,3,1)$ and 2 pairwise additive $\mathrm{B}\left(v^{\prime}, 3,1\right)$ are obtained by use of Theorems 3.4 and 4.4 and Lemma 6.1, and a $\operatorname{TD}(6, v)$ is also obtained by Lemma 6.3.

Hence on account of Lemma 6.6, the following result can be obtained. This improves Theorem 3.4.

Theorem 6.7 There are 2 pairwise additive $\mathrm{B}(6 m+1,3,1)$ for any positive integer $m$, except possibly for $6 m+1 \in\{55,115,145,205,265,319,355,415,493,667,697,1315\}$.

Unfortunately, we cannot clear such 12 values displayed in Theorem 6.7. Furthermore, the existence of 2 pairwise additive $\mathrm{B}(6 m+1,3,1)$ has not been known except for $6 m+3$ being $3^{n}$ and 15 in Theorem 4.4 and Lemma 6.1.

Remark. Since Theorem 4.2 can be valid for a given odd integer $k$, Theorem 5.6 is extended for a given odd integer $k$. On the other hand, when $k$ is an even prime power, an asymptotic existence of pairwise additive minimal $\mathrm{B}\left(v, 2^{n}, 2^{n}-1\right)$ is proved by some methods similar to Theorems 4.2, 4.3, 5.4 and 5.6. In particular, for $(\ell, n)=(2,1),(3,1)$, the complete existence of $\ell$ pairwise additive $\mathrm{B}(v, 2,1)$ has been shown in [4] [5]. How- 
ever, in general, the exact existence of $\ell$ pairwise additive minimal $\mathrm{B}(v, k, \lambda)$ with (1.1) could not be shown in this paper.

\section{References}

[1] Wilson, R.M. (1972) An Existence Theory for Pairwise Balanced Designs I. Journal of Combinatorial Theory, Series A, 13, 220-245. http://dx.doi.org/10.1016/0097-3165(72)90028-3

[2] Wilson, R.M. (1972) An Existence Theory for Pairwise Balanced Designs II. Journal of Combinatorial Theory, Series A, 13, 246-273. http://dx.doi.org/10.1016/0097-3165(72)90029-5

[3] Wilson, R.M. (1975) An Existence Theory for Pairwise Balanced Designs III. Journal of Combinatorial Theory, Series A, 18, 71-79. http://dx.doi.org/10.1016/0097-3165(75)90067-9

[4] Matsubara, K. and Kageyama, S. (2013) The Existence of Two Pairwise Additive $\operatorname{BIBD}(v, 2,1)$ for any $v$. Journal of Statistical Theory and Practice, 7, 783-790. http://dx.doi.org/10.1080/15598608.2013.783742

[5] Matsubara, K. and Kageyama, S. (to be Published) The Existence of 3 Pairwise Additive B( $v, 2,1)$ for Any $v \geq 6$. Journal of Combinatorial Mathematics and Combinatorial Computing.

[6] Matsubara, K., Sawa, M., Matsumoto, D., Kiyama, H. and Kageyama, S. (2006) An Addition Structure on Incidence Matrices of a BIB Design. Ars Combinatoria, 78, 113-122.

[7] Sawa, M., Matsubara, K., Matsumoto, D., Kiyama, H. and Kageyama, S. (2007) The Spectrum of Additive BIB Designs. Journal of Combinatorial Designs, 15, 235-254. http://dx.doi.org/10.1002/jcd.20147

[8] Sawa, M., Kageyama, S. and Jimbo, M. (2008) Compatibility of BIB Designs. Statistics and Applications, 6, 73-89.

[9] Mullin, R.C. and Gronau, H.D.O.F. (2007) PBDs and GDDs: The Basics. In: Colbourn, C.J. and Dinitz, J.H., Eds., The CRC Handbook of Combinatorial Designs, 2nd Edition, CRC Press, Boca Raton, 160-193.

[10] Raghavarao, D. (1988) Constructions and Combinatorial Problems in Design of Experiments. Dover, New York.

[11] Colbourn, C.J. and Ling, A.C.H. (1997) Pairwise Balanced Designs with Block Sizes 8, 9 and 10. Journal of Combinatorial Theory, Series A, 77, 228-245. http://dx.doi.org/10.1006/jcta.1997.2742

[12] Colbourn, C.J. and Rosa, A. (1999) Triple Systems. Oxford Press, New York, 404-406.

[13] Granville, A. (1988) Nested Steiner n-Cycle Systems and Perpendicular Arrays. Journal of Combinatorial Mathematics and Combinatorial Computing, 3, 163-167.

[14] Abel, R.J.R., Colbourn, C.J. and Dinitz, J.H. (2007) Mutually Orthogonal Latin Square. In: Colbourn, C.J. and Dinitz, J.H., Eds., The CRC Handbook of Combinatorial Designs, 2nd Edition, CRC Press, Boca Raton, 160-193. 
Scientific Research Publishing (SCIRP) is one of the largest Open Access journal publishers. It is currently publishing more than 200 open access, online, peer-reviewed journals covering a wide range of academic disciplines. SCIRP serves the worldwide academic communities and contributes to the progress and application of science with its publication.

Other selected journals from SCIRP are listed as below. Submit your manuscript to us via either submit@scirp.org or Online Submission Portal.
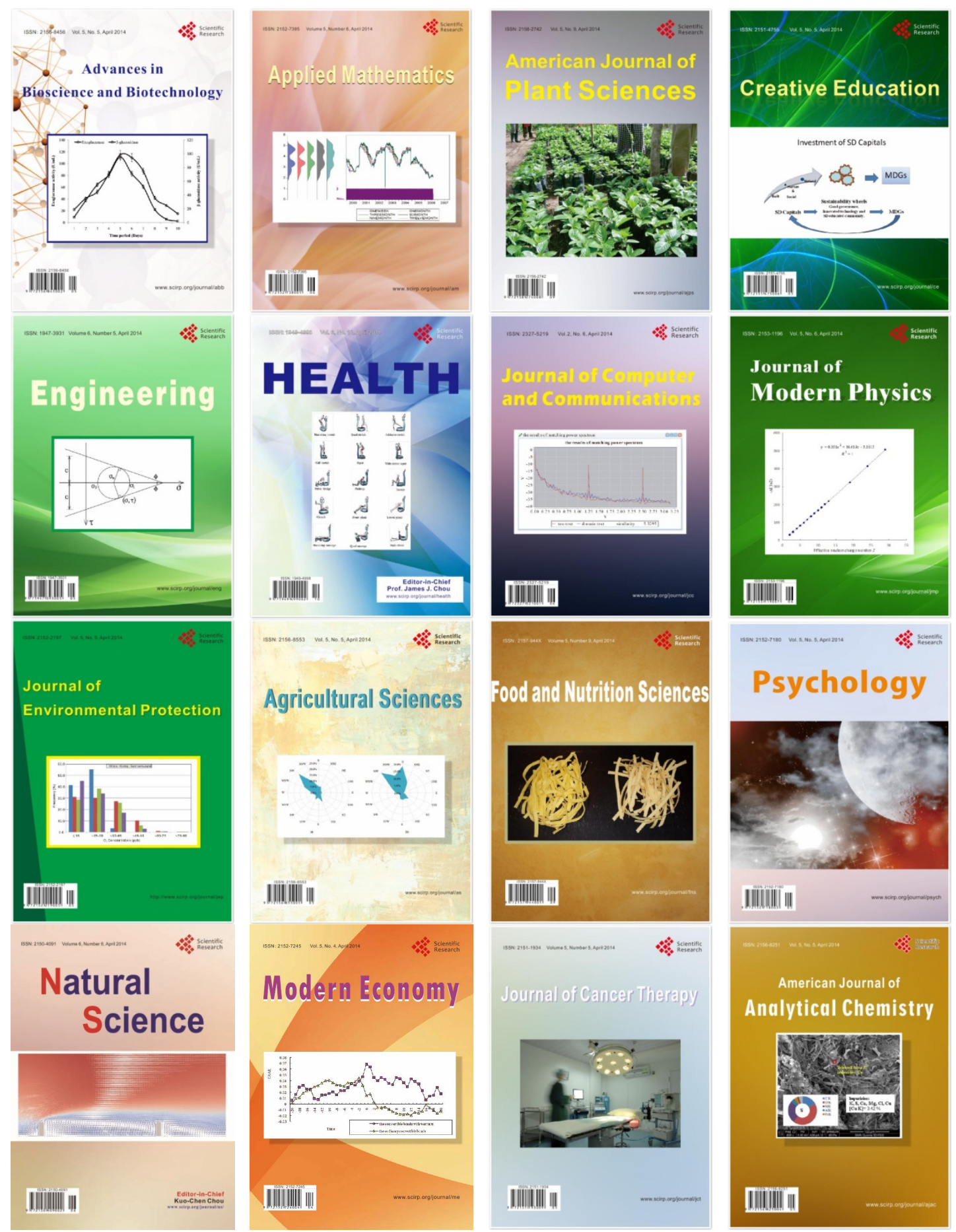